\title{
Clinical suppression of bradycardia dependent premature ventricular contractions by the potassium channel opener nicorandil
} Naohiko Takahashi, Morio Ito, Tetsunori Saikawa, Makoto Arita, Takehiko Fujino,
Hiroshi Kagiyama, Teruo Fukumoto, Toshiie Sakata
Department of Internal Medicine I, School of Medicine, Oita Medical University, Oita, Japan N Takahashi

T Saikawa

T Sakata

Department of Laboratory Medicine M Ito

Department of Physiology II M Arita

Department of Medicine, School of Medicine, Kyusyu University, Fukuoka, Japan

T Fujino

H Kagiyama

T Fukumoto

Correspondence to: Dr Takahashi, Department of Internal Medicine I, School of Medicine, Oita Medical University, 1-1 Idaigaoka, Hasama-machi, Oita 879-55, Japan.

Accepted for publication 1 September 1997

\begin{abstract}
Objective-To assess the clinical antiarrhythmic effect of nicorandil, a potassium channel opener, on premature ventricular contractions.

Design and patients-The effect of oral nicorandil (15 to $60 \mathrm{mg}$ daily for four weeks) on premature ventricular contractions was investigated in 20 patients (11 female, nine male, mean (SD) age 63 (17) years) who underwent 24 hour ambulatory ECG. Patients were classified into two groups based on the relation between the frequency of premature ventricular contractions and heart rate: (1) those with a positive correlation $(n=9)$; and (2) those with a bidirectional correlation $(n=11)$, characterised by an increased frequency of premature contractions at low heart rates and a decreased frequency at high heart rates.
\end{abstract}

Results-Nicorandil reduced the frequency of premature ventricular contractions by $75 \%$ in five patients in group 2 , but was not effective in any patient in group 1. The heart rate at which the frequency of premature ventricular contractions peaked was significantly lower in the five responders in group 2 than in the six non-responders $(63.2(3.7) v 76.3$ (12.4) beats/min, $\mathrm{p}<0.05)$.

Conclusions-Nicorandil may suppress premature ventricular contractions when they occur mainly at a low heart rate. (Heart 1998;79:64-68)

Keywords: nicorandil; premature ventricular contractions; early afterdepolarisations

Nicorandil is an antianginal drug with coronary vasodilator properties. ${ }^{1}$ Nicorandil activates the potassium channel in vascular smooth muscle, which partly explains its vasodilator actions. ${ }^{1}$ It also increases the membrane potassium conductance in cardiac muscles, causes hyperpolarisation, suppresses various kinds of automaticity, and lengthens the effective refractory period relative to the action potential duration. ${ }^{2-4}$ These actions are assumed to be beneficial in patients with ventricular arrhythmias. There are two case reports which described the efficacy of nicorandil on ventricular arrhythmias in patients with the idiopathic long QT syndrome. ${ }^{56}$

The frequency of premature ventricular contractions has been evaluated in previous studies as a function of the underlying heart rate using 24 hour ECG recordings. These studies showed interindividual variation in the relation between the frequency of premature ventricular contractions and heart rate. $^{7-9}$ In the present study we investigated the effect of nicorandil in patients with frequent premature ventricular contractions, using 24 hour ECG recordings, and we also examined the relation between the frequency of premature contractions and heart rate in these patients.

\section{Methods}

We studied 20 patients (nine men and 11 women) aged 19 to 91 years (mean (SD) age 63 (17) years) with frequent premature ventricular contractions (table 1), defined as more than 1000 premature contractions per 24 hours before the start of nicorandil treatment. Patients with sustained ventricular tachycardia were excluded. Informed consent was obtained from all patients.

Ten patients had cardiovascular disease, including hypertension in five, ischaemic heart disease in two, sick sinus syndrome in two, valvar disease in one, and dilated cardiomyopathy in one. The remaining 10 patients had no evidence of cardiac disease on the basis of their medical history, physical examination, 12 lead ECG, echocardiogram, and chest $x$ ray. Patients with an ejection fraction of less than $40 \%$, determined by echocardiography, were excluded.

All antiarrhythmic agents, including digitalis, were discontinued at least one week before any nicorandil was given. Nicorandil was given orally at a dose of 15 to $30 \mathrm{mg}$ daily for the first two weeks, and 15 to $60 \mathrm{mg}$ daily for the next two weeks. Ambulatory 24 hour ECG recordings were obtained before and four weeks after nicorandil treatment. Recordings were also obtained after two weeks of nicorandil treatment in 17 patients. Efficacy was defined as a $75 \%$ reduction in the frequency of premature ventricular contractions at the end of the four week treatment period. Premature ventricular contractions in individual patients were classified according to Lown's system as follows ${ }^{10}$ : class $2>30 / \mathrm{h}$; class 3 premature ventricular contractions of multiple origin; class $4 \mathrm{a}$ couplets; class $4 \mathrm{~b}$ triplets.

Ambulatory ECGs were recorded with a two channel Avionics recorder and analysed by an Avionics computer system (DCG 7 Dynamic Electroscanner, Avionics, Irvine, California, USA). This system automatically calculated 
Table 1 Clinical characteristics of the patients studied and findings on 24 hour ambulatory ECG

\begin{tabular}{|c|c|c|c|c|c|c|c|}
\hline $\begin{array}{l}\text { Case } \\
\text { No }\end{array}$ & Age/sex & $\begin{array}{l}\text { Cardiac } \\
\text { diagnosis }\end{array}$ & $\begin{array}{l}\text { Nicorandil } \\
(\text { mg/day })^{*}\end{array}$ & Control & $\begin{array}{l}\text { PVC/24 ht } \\
\text { at } 4 \text { weeks } \neq\end{array}$ & $\begin{array}{l}\text { Change } \\
(\%)\end{array}$ & Correlation\ \\
\hline 1 & $69 / \mathrm{F}$ & $\mathrm{AP}$ & $30-60$ & $10728(2)$ & $31210(2)$ & 191 & Positive \\
\hline 2 & $65 / \mathrm{F}$ & DCM & $30-60$ & $30504(4 b)$ & $54456(4 b)$ & 79 & Positive \\
\hline 3 & $50 / \mathrm{M}$ & $(-)$ & $30-60$ & 28848 & $22584(2)$ & -22 & Positive \\
\hline 4 & $73 / \mathrm{M}$ & HT & $30-45$ & $15960(4 b)$ & $6384(4 b)$ & -60 & Positive \\
\hline 5 & $91 / \mathrm{M}$ & OMI & $30-30$ & $3432(4 b)$ & $6240(4 b)$ & 82 & Positive \\
\hline 6 & $81 / \mathrm{F}$ & $\mathrm{AR}, \mathrm{MR}$ & $30-30$ & $13343(4 b)$ & $25152(4 b)$ & 88 & Positive \\
\hline 7 & $72 / \mathrm{M}$ & $(-)$ & $15-15$ & $2160(2)$ & $2927(2)$ & 36 & Positive \\
\hline 8 & $67 / \mathrm{M}$ & HT & $30-30$ & $2920(3)$ & $7056(4 b)$ & 142 & Positive \\
\hline 9 & $88 / \mathrm{F}$ & HT & $30-30$ & $54024(4 \mathrm{~b})$ & $35376(4 b)$ & -35 & Positive \\
\hline 10 & $54 / \mathrm{F}$ & $(-)$ & $30-45$ & $7874(2)$ & $8400(2)$ & 7 & Bidirectional \\
\hline 11 & $45 / \mathrm{F}$ & $(-)$ & $30-45$ & $6288(2)$ & $19608(2)$ & 212 & Bidirectional \\
\hline 12 & $57 / \mathrm{M}$ & $(-)$ & $30-30$ & $1872(2)$ & $2928(2)$ & 56 & Bidirectional \\
\hline 13 & $62 / \mathrm{M}$ & HT & $30-45$ & $5952(2)$ & $6816(2)$ & 15 & Bidirectional \\
\hline 14 & $19 / \mathrm{M}$ & $(-)$ & $30-30$ & $8160(2)$ & $7728(2)$ & -5 & Bidirectional \\
\hline 15 & $84 / \mathrm{F}$ & $(-)$ & $15-15$ & $1488(4 a)$ & $840(4 a)$ & -44 & Bidirectional \\
\hline 16 & $66 / \mathrm{F}$ & HT & $30-45$ & $11736(3)$ & $2520(2)$ & -79 & Bidirectional \\
\hline 17 & $46 / \mathrm{F}$ & SSS & $15-15$ & $19265(3)$ & 2357 (3) & -88 & Bidirectional \\
\hline 18 & $43 / \mathrm{F}$ & $(-)$ & $30-30$ & $13104(2)$ & $1416(2)$ & -89 & Bidirectional \\
\hline 19 & $61 / \mathrm{M}$ & SSS & $30-30$ & $2877(4 b)$ & $93(4 b)$ & -97 & Bidirectional \\
\hline 20 & $65 / \mathrm{F}$ & $(-)$ & $30-45$ & $3048(2)$ & 0 & -100 & Bidirectional \\
\hline
\end{tabular}

^Daily doses of nicorandil during the first 2 weeks and the next 2 weeks.

†Frequency of premature ventricular contractions (PVC) per 24 hours; $\ddagger 4$ weeks after nicorandil treatment.

CCorrelation between the frequency of PVC and heart rate before treatment. Lown's classification of PVC ${ }^{10}$ is given parentheses.

$\mathrm{AP}$, angina pectoris; AR, MR, aortic and mitral regurgitation; DCM, dilated cardiomyopathy; HT, hypertension; OMI, old myocardial infarction; SSS, sick sinus syndrome.

the total number of premature ventricular contractions during the 24 hour period and the mean daily heart rate. Data on the relation between the frequency of premature ventricular contractions were obtained using a computer system (M-343, Sord Compute System, Chiba, Japan) according to previously described methods. ${ }^{811}$ In brief, the heart rate and the frequency of premature ventricular contractions were determined every minute during the 24 hour period. The number of minutes at each heart rate (in one beat per minute increments) and the number of premature ventricular contractions at a given heart rate were determined. The frequency of premature ventricular contractions per minute was calculated for each heart rate from the formula: [number of premature ventricular contractions at a given heart rate]/[number of minutes at the same heart rate]. The frequency of premature ventricular contractions per minute was plotted against heart rate for all heart rates recorded for at least five minutes during the 24 hour period. The heart rate at which the frequency of premature ventricular contractions peaked was also determined.

We measured the QT interval on ECGs recorded between 0300 and 0400 and printed at a paper speed of $25 \mathrm{~mm} / \mathrm{s}$. We measured five consecutive RR and QT intervals. The QT interval was measured from the beginning of the QRS complex to the point at which the $T$ wave returned to the isoelectric line. The corrected QT interval (QTc) was calculated by Bazett's formula (QT interval in seconds divided by the square root of the RR interval in seconds), using the mean of the five measurements. ${ }^{12}$

DATA ANALYSIS

Data are expressed as mean values (SD), unless otherwise specified. Data were analysed by Student's $t$ test or Fisher's exact probability test; $\mathrm{p} \leqslant 0.05$ was considered significant.

\section{Results}

CORRELATION BETWEEN FREQUENCY OF PREMATURE VENTRICULAR CONTRACTIONS AND HEART RATE BEFORE TREATMENT

Patients showed one of two relations between the frequency of premature ventricular contractions and heart rate before treatment: either a positive correlation (group 1, $\mathrm{n}=9$; fig 1A), or a bidirectional correlation characterised by an increased frequency of premature ventricular contractions at relatively low heart rates and a decreased frequency at high heart rates (group 2, $\mathrm{n}=11$; fig $1 \mathrm{~B}-\mathrm{D}$ ).

EFFECTS OF NICORANDIL

Nicorandil reduced the frequency of premature ventricular contractions by $75 \%$ in five of the 11 patients with a bidirectional correlation (group 2) but in none of the nine patients in group 1, where there was a positive correlation between the frequency of premature ventricular contractions and heart rate (fig 2). The frequency of premature ventricular contractions and mean daily heart rate did not differ significantly before treatment between the responders and the non-responders in group 2 or the patients in group 1 (table 2). However, the heart rate at which the frequency of premature ventricular contractions peaked (arrows in fig 1) was significantly lower in the responders in group 2 than in the non-responders (table 2). The mean daily heart rate before nicorandil treatment was not significantly different from the rate after four weeks' treatment in any group.

Nicorandil altered Lown's classification in three patients (table 1): premature ventricular contractions of multiple origin improved to those of single origin in patient 3; couplets appeared after nicorandil treatment in patient 8 ; and premature ventricular contractions were completely abolished after nicorandil treatment in patient 2 . 


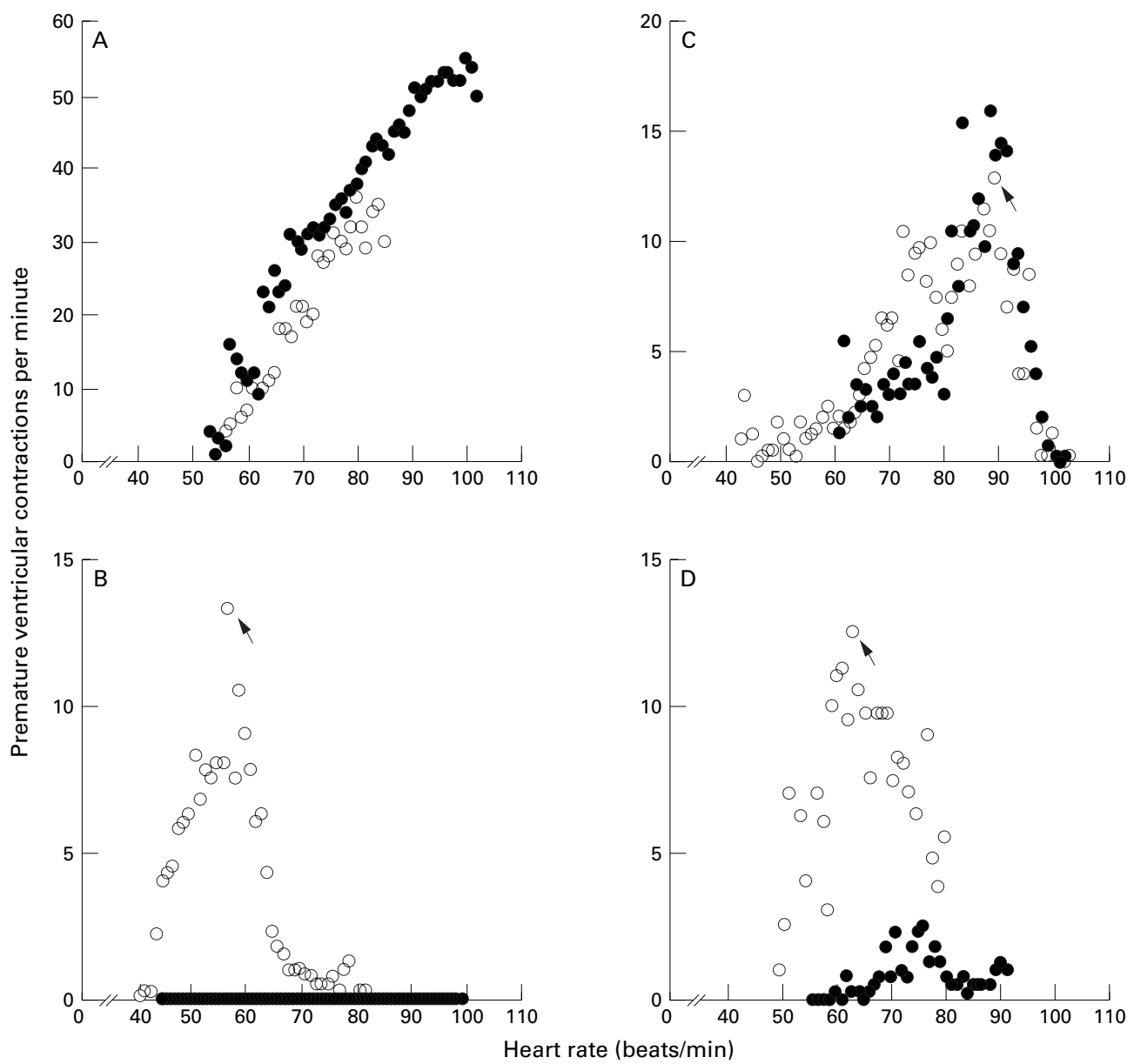

Figure 1 Relation between frequency of premature ventricular contractions (PVC) and heart rate in four patients before and four weeks after nicorandil treatment. Arrows indicate the heart rate at which the frequency of $P V C$ peaked (heart rate of peak PVC). (A) Patient showing a positive correlation between the frequency of PVC and heart rate. (B) Patient showing a bidirectional correlation. Nicorandil treatment caused negligible changes in these two patients. $(C)$ and $(D)$ Patients showing a bidirectional correlation between the frequency of $P V C$ and heart rate before nicorandil treatment. Nicorandil treatment caused a marked downward shift of the curve of the PVC frequency-heart rate relation. Note the lower heart rate of peak PVC in (C) and (D) compared with (B).

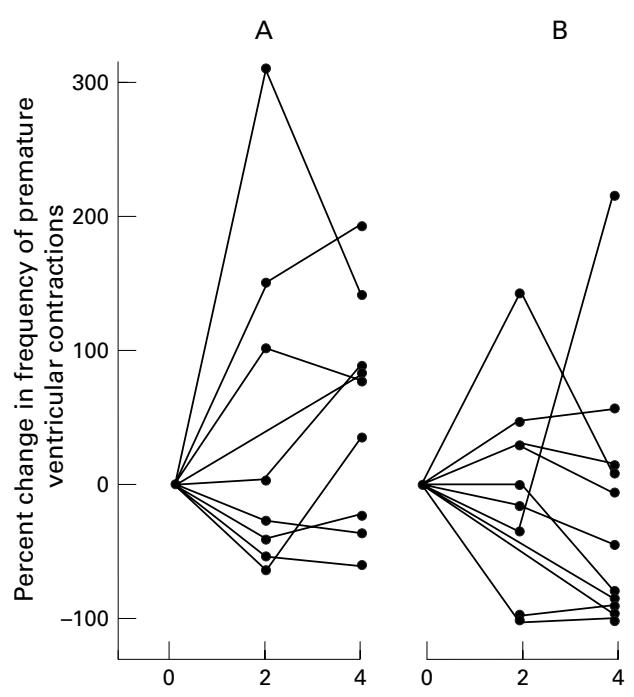

Duration of nicorandil treatment (weeks)

Figure 2 Percentage changes in the frequency of premature ventricular contractions (PVC) during nicorandil treatment. (A) Nine patients with a positive correlation between the frequency of $P V C$ and heart rate (group 1); (B) 11 patients with a bidirectional correlation (group 2).
QTC INTERVALS

The QTc intervals before treatment did not differ significantly among the groups. Nicorandil treatment caused no significant change in the QTc interval in any group: $0.40(0.02) v$ $0.41(0.03) \mathrm{ms}$ in group $1 ; 0.41(0.02) v$ $0.41(0.01) \mathrm{ms}$ in the non-responders in group 2 ; and $0.42(0.03) v 0.42(0.03) \mathrm{ms}$ in the responders in group 2.

\section{Discussion}

Nicorandil reduced the frequency of premature ventricular contractions by $75 \%$ in five of 20 patients $(25 \%)$, which is a lower efficacy than previously reported for other classic antiarrhythmic agents. ${ }^{13-15}$ Nicorandil was effective in five of the 11 patients who showed a bidirectional correlation between the frequency of premature ventricular contractions and heart rate (group 2), but in none of the patients with a positive correlation (group 1). The heart rate at which the frequency of premature ventricular contractions peaked was significantly lower in the five responders than in the six nonresponders in group 2 . The effect of nicorandil in this study differed from the effects of diltiazem and atenolol in our previous studies, 
Table 2 Comparison of 24 hour ECG findings in the three groups before treatment

\begin{tabular}{|c|c|c|c|}
\hline & \multirow[b]{2}{*}{$\begin{array}{l}\text { Positive group } \\
\text { (group 1) }\end{array}$} & \multicolumn{2}{|c|}{ Bidirectional group (group 2) } \\
\hline & & Non-responders & Responders \\
\hline Mean (SD) PVC/24h & $17991(17106)$ & $5268(2910)$ & $10006(7028)$ \\
\hline Mean (SD) heart rate (beats/min) & $75.1(14.3)$ & $71.3(6.3)$ & $69.2(4.4)$ \\
\hline $\begin{array}{l}\text { Mean (SD) heart rate of peak PVC† } \\
\text { (beats/min) }\end{array}$ & ND & $76.3(12.4)$ & $63.2(3.7)^{\star}$ \\
\hline
\end{tabular}

${ }^{\star} \mathrm{p}<0.05 v$ non-responder group.

†Heart rate at which the frequency of premature ventricular contractions (PVC) peaked

ND, not determined.
It is not possible to clarify the mechanism of premature ventricular contractions by analysing the relation between the frequency of premature contractions and the underlying heart rate. Imanishi $e t a l^{4}$ found that nicorandil suppresses both normal and abnormal automaticity, shortens the action potential duration, and prolongs the effective refractory period relative to the action potential duration in canine Purkinje fibres, suggesting that the suppression of premature ventricular contractions at high heart rates can be explained by overdrive suppression of automaticity or by the development of bidirectional block in the reentrant circuit. Further study is needed to clarify the mechanisms of the suppressive effect of nicorandil on bradycardia dependent premature ventricular contractions. findings suggest that nicorandil may be effective in certain patients whose premature ventricular contractions occur mainly at lower heart rates.

The suppressive effect of nicorandil on bradycardia dependent premature ventricular contractions may involve various mechanisms. Nicorandil activates the potassium channel and the cyclic guanosine monophosphate dependent pathway of vascular smooth muscle, and thus has potent vasodilator effects. ${ }^{1}$ While there was no evidence of manifest ischaemic heart disease in the five responders, the possibility that nicorandil suppressed premature ventricular contractions by reducing myocardial ischaemia cannot be excluded.

The nicorandil induced activation of the potassium channel of the cardiac membrane $\mathrm{e}^{2-4}$ may contribute to the suppression of bradycardia dependent premature ventricular contractions. Torsade de pointes in patients with the long QT syndrome tends to occur when the potassium conductance of the cardiac membrane is decreased, and is promoted by bradycardia. ${ }^{16}$ The mechanism of torsade de pointes has been attributed to early afterdepolarisation. ${ }^{16}$ We previously reported that nicorandil suppressed early afterdepolarisations and ventricular arrhythmias induced by caesium chloride in rabbits. ${ }^{17}$ Pinacidil and cromakalim, other potassium channel openers, were also found to be effective at abolishing early afterdepolarisations and related ventricular arrhythmias in animal studies. ${ }^{18} 19$ These observations suggest that potassium channel openers suppress ventricular arrhythmias in patients with the long QT syndrome. ${ }^{20}$ In fact, two case reports have documented the abolition of early afterdepolarisations and torsade de pointes in patients with the idiopathic long QT syndrome by nicorandil treatment. ${ }^{56}$ In the present study, none of the patients had abnormal QT prolongation before treatment, and nicorandil treatment did not affect the QTc interval in any patient. However, the suppression of premature ventricular contractions observed in the five responders to nicorandil may have been due to an enhanced cardiac potassium conductance because early afterdepolarisations in a limited area of the ventricle do not lead to abnormal QT prolongation on the surface ECG. ${ }^{21}$

\section{LIMITATIONS}

We studied a relatively small number of patients. There was also considerable variation in the day to day frequency of premature ventricular contractions in the absence of antiarrhythmic drugs. ${ }^{11}$ Thus it is possible that the nicorandil induced reduction in the frequency pf premature ventricular contractions in the five responders was due to the day to day variation. However, it is unlikely that such a spontaneous reduction occurred only in patients with bradycardia dependent premature ventricular contractions. Another limitation is that we assessed efficacy in terms of the reduction in the number of premature ventricular contractions. Lown's classification was not improved in four of the five responders. It remains to be determined whether the reduction in the number of premature ventricular contractions produced by nicorandil was beneficial to the patients. ${ }^{22}$

This study was supported in part by Chugai Pharmaceutical Co (Tokyo, Japan).

1 Furukawa K, Itoh T, Kajiwara M, Kitamura K, Suzuki H, Ito $\mathrm{Y}$, et al. Vasodilating actions of 2-nicotinamidoethyl nitrate on porcine and guinea-pig coronary arteries. $\mathcal{F}$ Pharmacol Exp Ther 1981;218:248-59.

2 Yanagisawa T, Taira N. Effect of 2-nicotinamidethyl nitrate (SG-75) on the membrane potential of left atrial muscle fibres of the dog. Increase in potassium conductance. Naunyn Schmiedebergs Arch Pharmacol 1980;312:69-76.

3 Hiraoka M, Fan Z. Activation of ATP-sensitive outward K+ isolated ventricular myocytes. F Pharmacol Exp Ther 1989; 250:278-85.

4 Imanishi S, Arita M, Aomine M, Kiyosue T. Antiarrhythmic effects of nicorandil on canine cardiac Purkinje fibers. $\mathcal{F}$ Cardiovasc Pharmacol 1984;6:772-9.

5 Sato T, Hata Y, Yamamoto $M$, Morita $H$, Mizuo K, Yamanari H, et al. Early afterdepolarization abolished by potassium channel opener in a patient with idiopathic long QT syndrome. F Cardiovasc Electrophysiol 1995;6:279-82.

QT syndrome. F Cardiovasc Electrophysiol 1995;6:279-82. Chinushi M, Aizawa Y, Furushima H, Inuzuka H, Ojima K, sic action potential and torsade de pointes in a patient with idiopathic long QT syndrome. Jpn Heart f 1995;36:47781 . beat frequency and heart rate. Circulation 1982;66:439-46.

8 Ito M, Tsumabuki S, Maeda Y, Arita M, Saikawa T, Ito S, et al. Suppression of ventricular premature contractions posatenolol. fpn Circ f 1987;51:217-29.

9 Ito M, Maeda Y, Arita M, Ito S, Saikawa T, Omura I, et al. Effects of oral diltiazem on ventricular premature contractions. F Electrocardiol 1986;19:59-66.

10 Lown B, Wolf $M$. Approaches to sudden death from coronary heart disease. Circulation 1971;44:130-42.

11 Tsumabuki S, Ito M, Arita M, Saikawa T, Ito S. Day-to-day variation of the frequency of ventricular premature contractions depends on variation of heart rates. Fpn Circ $\mathcal{F}$ 1988;52:1231-9. current by nicorandil (2-nicotinamidoethyl nitrate) in

7 Winkle RA. The relationship between ventricular ectopic sibly related to triggered activity by oral diltiazem and 
12 Bazett HC. An analysis of the time-relations of electrocardiogram. Heart 1920;7:353-70.

13 Morganroth J. Ambulatory electrocardiographic monitoring in the evaluation of new antiarrhythmic drugs. Circulation pl II):II-92-7.

14 Winkle RA, Gradman AH, Fitzgerald JW. Antiarrhythmic drug effect assessed from ventricular arrhythmia reduction in the ambulatory electrocardiogram and treadmill test: comparison of propranolol, procainamide and quinidine. Am $\mathcal{F}$ Cardiol 1978;42:473-80.

15 Salerno DM, Gillingham KJ, Berry DA, Hodges M. A comparison of antiarrhythmic drugs for the suppression of ventricular ectopic depolarizations: a meta-analysis. Am Heart f 1990;120:340-53.

16 Jackman WM, Friday KJ, Anderson JL, Aliot EM, Clark M, Lazzara R. The long QT syndromes: a critical review. New clinical observations and a unifying hypothesis. Prog Cardiovasc Dis 1988;31:115-72.

17 Surawicz B. Electrophysiologic substrate of torsade de pointes: dispersion of repolarization or early afterdepolarizations? $\mathcal{f}$ Am Coll Cardiol 1989;14:172-84.
18 Takahashi $N$, Ito $M$, Saikawa $T$, Arita $M$. Nicorandil suppresses early afterdepolarisation and ventricular arrhythmias induced by caesium chloride in rabbits in vivo. Cardiovasc Res 1991;25:445-52.

19 Fish FA, Prakash C, Roden DM. Suppression of repolarization-related arrhythmias in vitro and in vivo by low-dose potassium channel activators. Circulation 1990; 82:1362-9.

20 Carlsson L, Abrahamsson C, Drews L, Duker G. Antiarrhythmic effects of potassium channel openers in tiarrhythmic effects of potassium channel openers in rhythm abnormalities related
Circulation 1992;85:1491-500.

21 Locati EH, Schwartz PJ. The idiopathic long QT syndrome: therapeutic management. PACE 1992;15: $1374-9$.

22 Echt DS, Liebson PR, Mitchell LB, Peters RW, ObiasManno D, Barker AH, et al. Mortality and morbidity in patients receiving encainide, flecainide, or placebo. The Cardiac Arrhythmia Suppression Trial. $N$ Engl $f \mathrm{Med}$ 1991;324:781-8. 